\title{
Magnetic Assembly and Pyrolysis of Functional Ferromagnetic Colloids into One-Dimensional Carbon Nanostructures
}

\author{
Steven E. Bowles, ${ }^{\dagger}$ Wei Wu, ${ }^{\S}$ Tomasz Kowalewski, ${ }^{\S}$ Matthew C. Schalnat, ${ }^{\dagger}$ Robert J. \\ Davis, ${ }^{\dagger}$ Jeanne E. Pemberton, ${ }^{\dagger}$ Inbo Shim, ${ }^{\dagger \dagger}$ Bryan D. Korth, ${ }^{\dagger}$ and Jeffrey Pyun ${ }^{\dagger *}$ \\ ${ }^{\dagger}$ Department of Chemistry, University of Arizona, 1306 E. University Blvd. Tucson, AZ 85752, \\ ${ }^{\ddagger}$ Department of Nano and Electronic Physics, Kookmin University, Seoul, Korea, 136-702, \\ ${ }^{\S}$ Department of Chemistry, Carnegie Mellon University, 4400 Fifth Avenue, Pittsburgh, PA 15213
}

email: jpyun@email.arizona.edu

\section{Supporting Information}

\section{TABLE OF CONTENTS}

\section{Experimental}

II. Synthesis of PAN Encapsulated Nanoparticles and 1-D Carbon Nanoparticle Chains III. Solid State Characterization of Nanocomposite Compositions (XRD, DT-TGA, XPS, Raman)

IV. AFM/FESEM/TEM images

V. Magnetic Properties (VSM)

VI. References for Supporting Information and Manuscript

\section{Experimental}

Materials and Characterization. Tetrahydrofuran (THF), neutral alumina, methanol (MeOH), magnesium sulfate, ethylene carbonate, trifluoroacetic acid, N,N-dimethylformamide (DMF), ethyl ether, ethanol, and 2,2-dipyridyl were purchased from Aldrich and used as received without further purification. Dicobaltoctacarbonyl $\left(\mathrm{Co}_{2}(\mathrm{CO})_{8}\right)$ was purchased from Strem and stored in a desiccator at $0^{\circ} \mathrm{C}$. Copper(I) bromide was purchased from Aldrich and purified by stirring in glacial acetic, filtering, washing with ethanol and ethyl ether, and drying under vacuum before use. Acrylonitrile was purchased from Aldrich and passed through a short column of neutral alumina prior to use. Nuclear magnetic resonance (NMR) was performed using a Bruker DRX 250 MHz FT-NMR spectrometer, operating XWinNMR software (Bruker). Size exclusion chromatography of polyacrylonitrile was performed using DMF as an eluent with a Waters 510 
HPLC pump, three Waters Ultrastyragel columns (500,10 $0^{3}$ and $\left.10^{5} \AA\right)$, and a Waters 410 DRI detector. A flow rate of $1.0 \mathrm{~mL} / \mathrm{min}$ was used, calibrated with polystyrene standards.

DT-TGA measurements was carried out using a DT-TGA Q50 (TA Instruments) instrument and software from TA Instruments. TGA measurements were taken from $20^{\circ} \mathrm{C}$ to $1000^{\circ} \mathrm{C}$ at a ramp rate of $10^{\circ} \mathrm{C}$ per minute. TEM images were obtained on a JEM100CX II transition electron microscope (JEOL) at an operating voltage of $60 \mathrm{kV}$, using in house prepared nickel grids (Ni, square, 300 mesh). Analysis of images was carried out using custom programs written in Matlab 7.0. FE-SEM images were taken on a Hitachi S-4500 Field Emission Scanning Electron Microscope at an operating voltage of 1-10 kV on silicon wafers (Wafer World Inc, West Palm Beach, FL). VSM measurements were obtained using a Waker HF 9H electromagnet with a Lakeshore 7300 controller and a Lakeshore 668 power supply. Magnetic measurements were carried out at room temperature $(300 \mathrm{~K})$ and low temperature $(40 \mathrm{~K})$, with a maximum applied field of $15 \mathrm{kOe}$, a ramp rate of $33 \mathrm{Oe} / \mathrm{s}$ and a time constant of 0.1 . XRD measurements were performed using the X'pert X-ray diffractometer (PW1827) (Phillips) at room temperature with a CuKa radiation source at $40 \mathrm{kV}$ and $30 \mathrm{~mA}$. The scan angle was from 30 to 80 degrees with a scan size of 0.2 degrees and a scan time of 0.5 seconds per 0.2 degrees. XPS characterization was performed on silicon wafer using a KRATOS 165 Ultra photoelectron spectrometer, using a monochromatic Al Ka radiation source. Substrates for Raman spectral analysis of these films were composed of quartz microscope slides (GM Associates, Oakland, CA). Raman spectroscopy was carried out at room temperature using $50 \mathrm{~mW}$ of $700 \mathrm{nnm}$ radiation at the sample from a Ti:sapphine laser (Spectra Physics 3900S CW ) pumped by an $\mathrm{Ar}^{+}$ laser (Coherent Innova 350C.) Scattered photons were collected at an angle of $30^{\circ}$ with respect to the surface normal using a single monochromator (SPEX 270M) with a $100 \mu \mathrm{m}$ entrance slit and a 1340 x 400 EB back illuminated CCD (Roper Scientific, operating temperature of $-95^{\circ} \mathrm{C}$.) A holographic Super Notch Plus filter (Kaiser Optical Systems) was used to reduce Rayleigh scattering. Typical integration times varied from 1-10 minutes per acquisition and spectra were calibrated using the Raman-active modes of a monolayer of thiophenol chemisorbed on Au.

Tapping mode atomic force microscopy (TMAFM). Tapping mode AFM experiments were carried out using a Multimode Nanoscope III system (Digital Instruments, Santa Barbara, CA). The measurements were performed in air using commercial Si cantilevers with a nominal spring constant and resonance frequency respectively equal to $40 \mathrm{~N} / \mathrm{m}$ and $300 \mathrm{kHz}$. The height and phase images were acquired simultaneously at set-point ratio $A / A_{o}=0.7 \sim 0.9$, where $A$ and $A_{o}$ refer to the "tapping" and "free" cantilever amplitude, respectively. The TMAFM images were analyzed quantitatively using custom procedures written in MATLAB 7.0 (Mathworks, Inc., 
Natick, MA). The high-pass filtering of zoomed-in images shown in the right column of Figure 2 was performed by taking the first derivative of height image along the y-axis. Such unidirectional filtering enhanced the visualization of boundaries between the individual particles comprising aligned chain nanostructures.

\section{Synthesis of PAN Encapsulated Nanoparticles and 1-D Carbon Nanoparticle Chains Synthesis of polyacrylonitrile.}

The general strategy that was developed to prepare 1-D carbon nanoparticle chains, was the synthesis of PAN surfactants via ATRP, ligand exchange onto cobalt nanoparticles and pyrolysis of assemblied PAN-CoNP chains. The ligand exchange of PS with PAN was derived from literature precedence relying on a high affinity binding group (nitrile) and a statistical excess of the exchange polymer (5:1 PAN/PS). Extensive research on the functionalization of gold, iron, and semiconductor nanoparticles, via ligand exchange, has demonstrated the viability of using polar ligating groups, such as thiols, carboxylic acids, diols, and phenols, to coordinate polymers onto magnetic nanoparticles.[1-3] In the patent literature, ligand exchange was reported for cobalt nanoparticles bound with P(MMA-r-EA-r-VPy) surfactants and exchanged with other copolymers possessing polar side chain groups. [4] Nitrile containing block copolymers, based of polysiloxanes and polystyrenics, were used to synthesize cobalt nanoparticles, and the nitrile group ensured that nanoparticles remained colloidally stable. $[5,6]$

Synthesis of polyacrylonitrile using ATRP. Synthesis of PAN surfactants was conducted using reported methods. [7] To a Schlenk flask was added copper(I) bromide (0.054 g, $0.379 \mathrm{mmol})$, 2,2-dipyridyl (0.118 g, $0.759 \mathrm{mmol})$, and ethylene carbonate $(12 \mathrm{~g})$, and the flask was placed under vacuum for 30 minutes. The flask was heated to $40^{\circ} \mathrm{C}$ to melt the ethylene carbonate, and the flask was backfilled with argon. A solution of acrylonitrile $(5.0 \mathrm{~mL}, 75.9 \mathrm{mmol})$ and methyl 2-bromopropionate $(0.045 \mathrm{~mL}, 0.379 \mathrm{mmol})$ was degassed by argon bubbling for 30 minutes, added via syringe into the Schlenk flask, and heated with stirring at $55^{\circ} \mathrm{C}$ for 90 minutes. The viscous solution was removed from heat, precipitated into THF $(300 \mathrm{~mL})$, and cooled to $0^{\circ} \mathrm{C}$ for 20 minutes. The precipitate was collected by vacuum filtration, and washed with $\mathrm{MeOH}$. The light blue solid was resuspended in $\mathrm{MeOH}$, sonicated, and filtered to remove residual copper. The off-white solid was dried under vacuum to constant weight $\left(1.34 \mathrm{~g}, \mathrm{M}_{\mathrm{n}}=21,800, \mathrm{M}_{\mathrm{w}} / \mathrm{M}_{\mathrm{n}}=\right.$ 1.13, 70.7 \%). ${ }^{1} \mathrm{H}$ NMR (250 MHz, d6-DMSO): $\delta 3.4-3.0$ (bs, -CHCN-), $\delta 2.4-1.9$ (bs, $\left.-\mathrm{CH}_{2}-\right), \delta$ $1.183\left(\mathrm{~d}, 3 \mathrm{H},-\mathrm{CH}_{3}\right)$. 
Synthesis of PS-Co Nanoparticles. The synthesis of ferromagnetic polystyrene coated nanoparticles was conducted using reported methods. [8]

PAN-Co Nanoparticles via Ligand Exchange. A solution of PS-Co nanoparticles was prepared by sonicating PS-Co nanoparticles $(0.302 \mathrm{~g}, 20 \mathrm{wt} \%$ polymer/ $80 \mathrm{wt} \%$ cobalt by TGA)[8] in DMF ( $40 \mathrm{~mL})$ for 5 minutes. To the nanoparticle solution was added PAN $(0.310 \mathrm{~g})$, followed by sonication at $50^{\circ} \mathrm{C}$ for 3 hours. The solution was diluted with THF $(45 \mathrm{~mL})$ and centrifuged twice at $7500 \mathrm{rpm}$ for 30 minutes. After decanting of the supernatant, the pellet was transferred with acetonitrile and dried under vacuum for 2.5 hours to constant weight $(0.214 \mathrm{~g})$. Polymer shell characterization was verified by degrading the particles with TFA and analyzing the organic residue by SEC and NMR (see next section). Bulk composition of the PAN cobalt nanoparticles was determined by XRD (Figure S-1), DT-TGA (Figure S-2), XPS (Figure S-3,4), and RAMAN (Figure S-5). Particle diameter, size distributions, and magnetic properties were determined by AFM (Figure S-6,7,8), TEM (Figure S-10), FESEM (Figure S-11,12,13,14,15,16), and VSM (Figure S-17,18).

Degradation of PAN cobalt nanoparticles for analysis of polymer shell. The polymer shell composition of the PAN cobalt (PAN-Co) nanoparticles was identified by degrading the cobalt nanoparticles with TFA and analyzing the residual polymer by ${ }^{1} \mathrm{H}-\mathrm{NMR}$ and SEC. For ${ }^{1} \mathrm{H}-\mathrm{NMR}$ characterization, a solution of PAN-Co nanoparticles $\left(11.6 \mathrm{mg}\right.$ ) was dispersed in $d_{6}$-DMSO (400 $\mathrm{mg}$ ) by sonication. To the PAN-Co nanoparticles solution was added TFA (25 mg). The solution was sonicated for 1 hour. After sonication, a color change from black to purple was observed. The sample was neutralized with $\mathrm{K}_{2} \mathrm{CO}_{3}$, dried with $\mathrm{MgSO}_{4}$, and filtered through alumina. ${ }^{1} \mathrm{H}$ NMR of the sample showed no residual polystyrene peaks. ${ }^{1} \mathrm{H}$ NMR $\left(250 \mathrm{MHz}, d_{6}\right.$-DMSO): $\delta$ 3.4-3.0 (bs, -CHCN-), $\delta$ 2.4-1.9 (bs, $-\mathrm{CH}_{2}$ ). For SEC characterization, a solution of PAN-Co nanoparticles $(10.8 \mathrm{mg}$ ) was dispersed in DMF $(515 \mathrm{mg})$ by sonication. To the PAN-Co nanoparticles solution was added TFA $(25.2 \mathrm{mg})$, followed by brief heating with a heat gun for 5 seconds and sonication for 30 minutes. After sonication, a color change from black to purple was observed. The sample was neutralized with $\mathrm{K}_{2} \mathrm{CO}_{3}$, dried with $\mathrm{MgSO}_{4}$, and filtered through alumina. SEC-DMF of the sample corresponded to pre-exchanged PAN $\left(M_{n}=22,600, M_{w} / M_{n}=\right.$ 1.14) with no residual polystyrene.

Typical pyrolysis conditions of the PAN-Co nanoparticles. Pyrolysis of the PAN-Co nanoparticles was performed in a two step process in a TGA or tube furnace.[9] The PAN-Co 
nanoparticles were stabilized by heating in air at $250^{\circ} \mathrm{C}$ for 2 hours. After cooling back to room temperature, the stabilized PAN-Co nanoparticles were heated under inert atmosphere (argon) to $600^{\circ} \mathrm{C}$ at $20^{\circ} \mathrm{C} / \mathrm{min}$ and maintained at $600^{\circ} \mathrm{C}$ for 2 hours. Samples were cooled to RT under argon.

\section{Solid State Characterization of PAN-Co Nanoparticle Compositions (XRD, DT-TGA, XPS, RAMAN)}

Solid State Characterization of PAN-Co nanoparticles by XRD. The crystalline phase of the polymer coated nanoparticles was determined to be fcc by XRD before and after pyrolysis (Figure S-1). The peaks of the pre-pyrolysized PAN-Co nanoparticles correspond with simulated XRD peaks for fcc cobalt nanoparticles, in agreement with previous reports of cobalt nanoparticle preparation from PS N-functionalized surfactant systems.[10]'[8]'[4] Pyrolysis was performed on a bulk sample of PAN cobalt nanoparticles $(35 \mathrm{mg})$ by typical procedure in a tube furnace. The crystalline phase of the pyrolysized PAN-Co nanoparticles was determined to be primarily fcc with trace amounts of hcp. The fcc (111) peak at 44 degree was sharper, due to annealing of the cobalt at $600^{\circ} \mathrm{C}$.

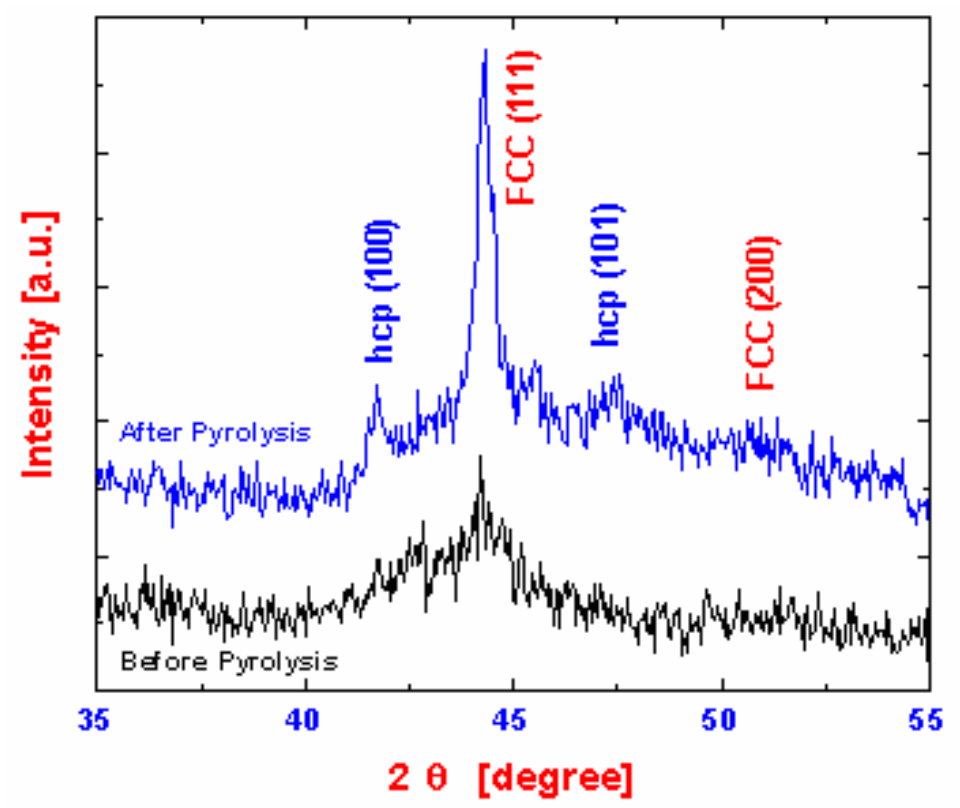

Figure S-1. XRD spectra of fcc-phase PAN-Co nanoparticles before and after pyrolysis.

Solid State Characterization of PAN-Co nanoparticles by DT-TGA. Interrogation of the polymer shell surrounding the cobalt nanoparticle core was obtained via DT-TGA measurements. 
The amount of polymer incorporated during the polymer exchange reaction was determined to be approximately $40 \%$ of the total mass, as indicated by the loss of mass between $300-400^{\circ} \mathrm{C}$ (Figure S-2). The loss of mass at approximately $100^{\circ} \mathrm{C}$ was attributed to residual solvent $(2.224 \%)$.

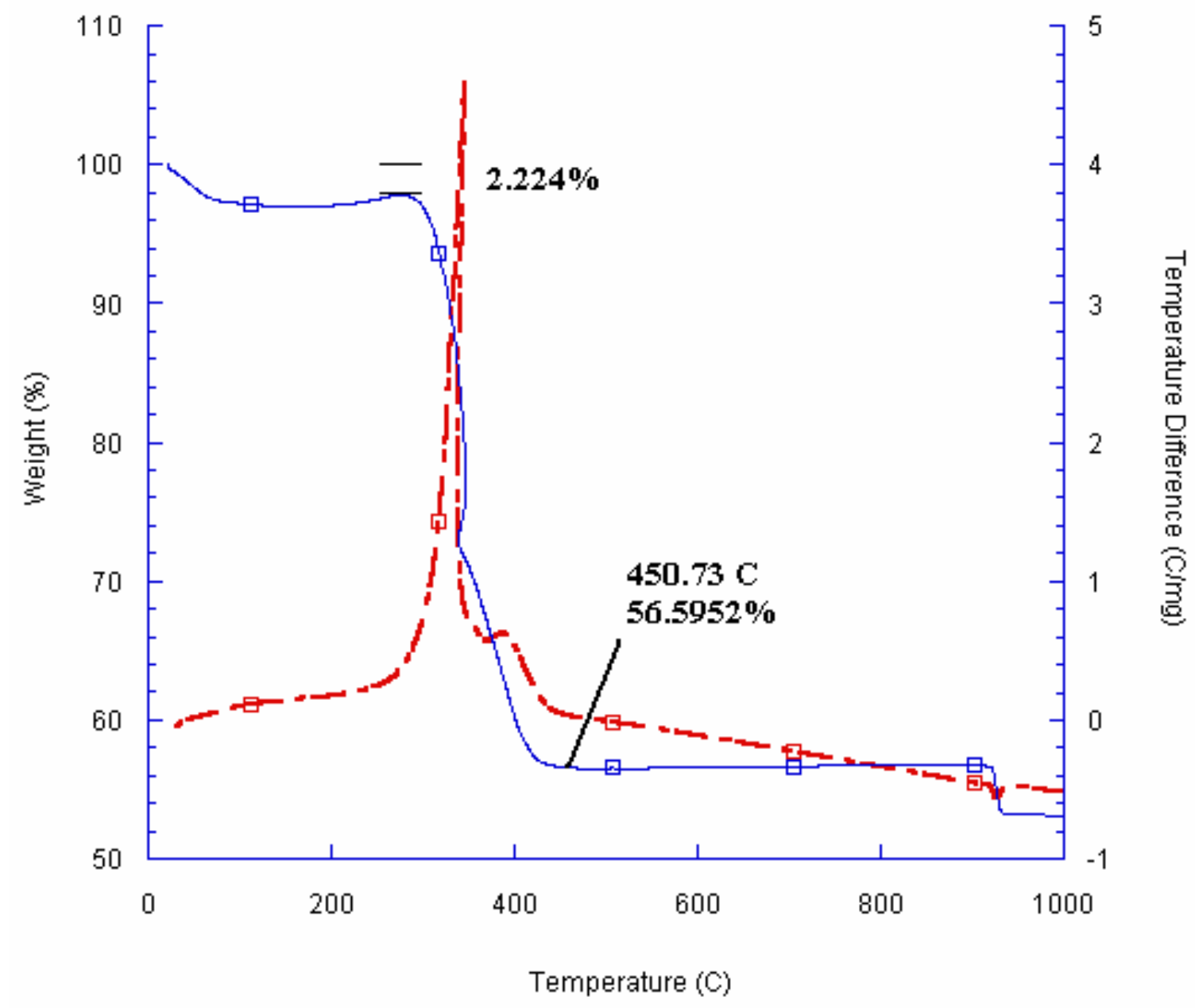

Figure S-2: DT-TGA graph of PAN-Co nanoparticles

Solid State Characterization of PAN-Co nanoparticles by XPS. The presence of both PAN and metallic cobalt was confirmed using XPS on thin films of nanocomposite materials on Si wafers. A solution of PAN-Co nanoparticles in DMF (10 mg/mL solution) was sonicated, drop cast onto $\mathrm{Si}$ wafer, and dried in a magnetic field. Before pyrolysis, peaks at $286 \mathrm{eV}$ and $401 \mathrm{eV}$ were assigned to $\mathrm{C} 1 \mathrm{~s}$ and N1s peaks present in PAN (Figure S-3). XPS revealed the presence of oxygen $(535 \mathrm{eV})$, cobalt (II) $(780 \mathrm{eV})$ and cobalt $(0)(776 \mathrm{eV})$ species, which confirmed the formation of metallic cobalt and cobalt oxide $(\mathrm{CoO})$. The limited penetration depth of XPS ( few nanometers), inflated the contribution of $\mathrm{CoO}$ shells in the XPS spectrum, but nevertheless 
confirmed the formation of a PAN-cobalt nanocomposite material, in agreement with AFM and XRD data.[8]
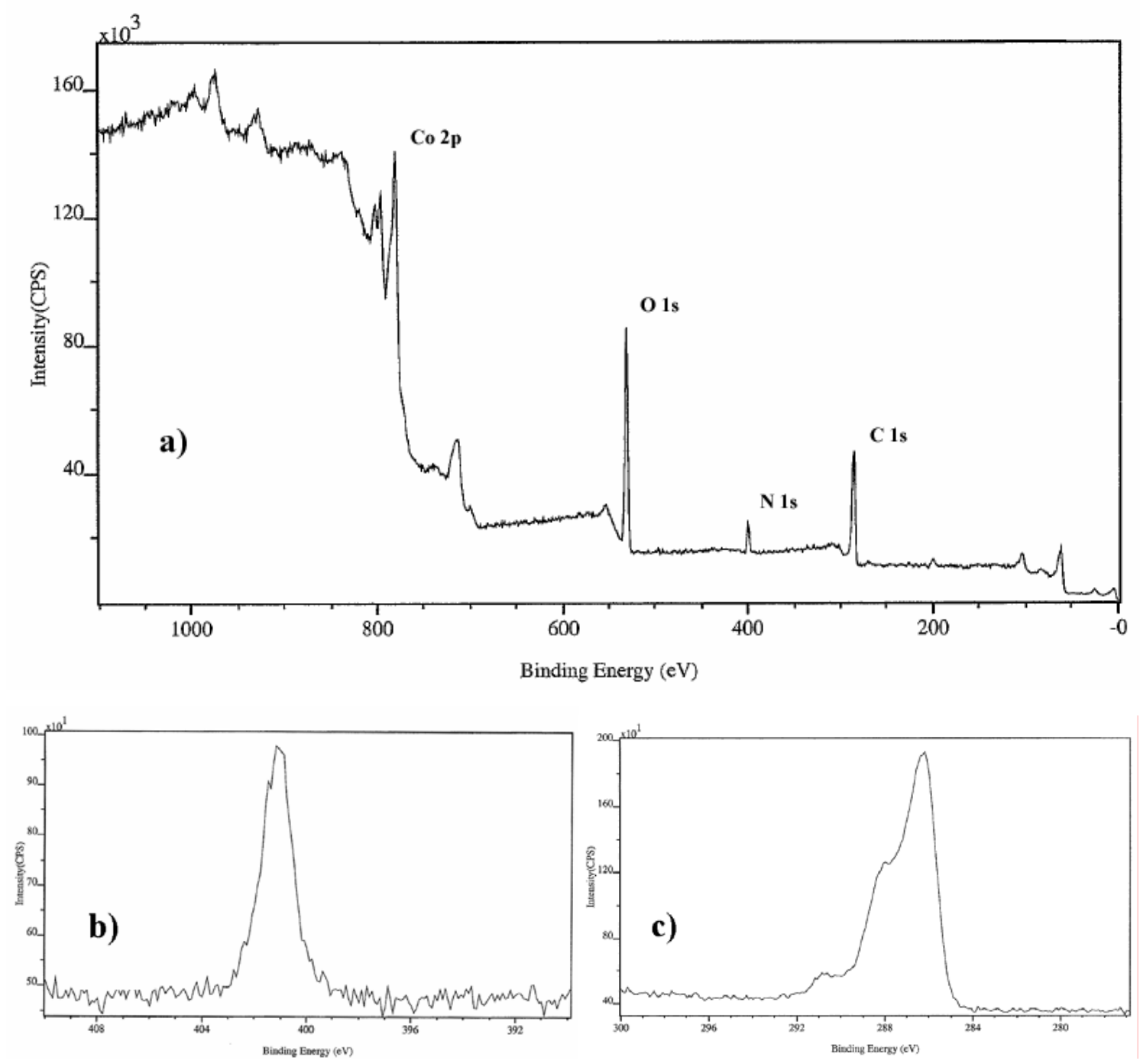

Figure S-3: (a) XPS spectrum of PAN Co nanoparticles before pyrolysis (b) expanded spectrum of nitrogen peaks (c) expanded spectrum of carbon peaks

Pyrolysis of the PAN Co to graphitic carbon Co nanoparticles was done in a TGA on Si wafer using typical pyrolysis conditions. Peaks at $535 \mathrm{eV}$ (O 1s), $780 \mathrm{eV}$ (Co (II) 2p) and $776 \mathrm{eV}$ (Co (0) 2 p) were present, confirming the retention of metallic cobalt and cobalt oxide (Figure S-4a). The observed changes to the N 1s and C 1s peak were consistent with literature XPS studies on pyrolysis of free PAN films, noting that the cobalt inclusions catalyzed the carbonization of the PAN. The XPS N $1 \mathrm{~s}$ and C 1 s peak of the pyrolysized PAN- Co nanoparticles at $600^{\circ} \mathrm{C}$ were consistent with the pyrolyzed free PAN at $1100^{\circ}$ C.[11] The $\mathrm{N} 1$ s peak at $401 \mathrm{eV}$ decreased in intensity consistent with the high temperature $1100^{\circ} \mathrm{C}$ pyrolysis of free PAN (Figure S-4b). The 
$\mathrm{C} 1 \mathrm{~s}$ peak at $286 \mathrm{eV}$ decreased in intensity, which was expected due to loss of mass during pyrolysis. The population of different types of $\mathrm{C}$ bonds shifted from a peak with two shoulders before pyrolysis, representing the populations of $\mathrm{C}=\mathrm{C} \mathrm{sp}^{2}$ bonds, $\mathrm{C}=\mathrm{N} \mathrm{sp}{ }^{2}$ bonds, and $\mathrm{C}=\mathrm{O} \mathrm{sp}^{2}$ bonds, to a single peak after pyrolysis, representing the $\mathrm{C}=\mathrm{C} \mathrm{sp}{ }^{2}$ bonds (Figure $\mathrm{S}-4 \mathrm{~b}$ ). The catalytic effect of cobalt in the pyrolysis step has been described in the literature regarding the conversion of diamond nanoparticles to graphite coating on cobalt nanoparticles at $600^{\circ} \mathrm{C}$.[12]
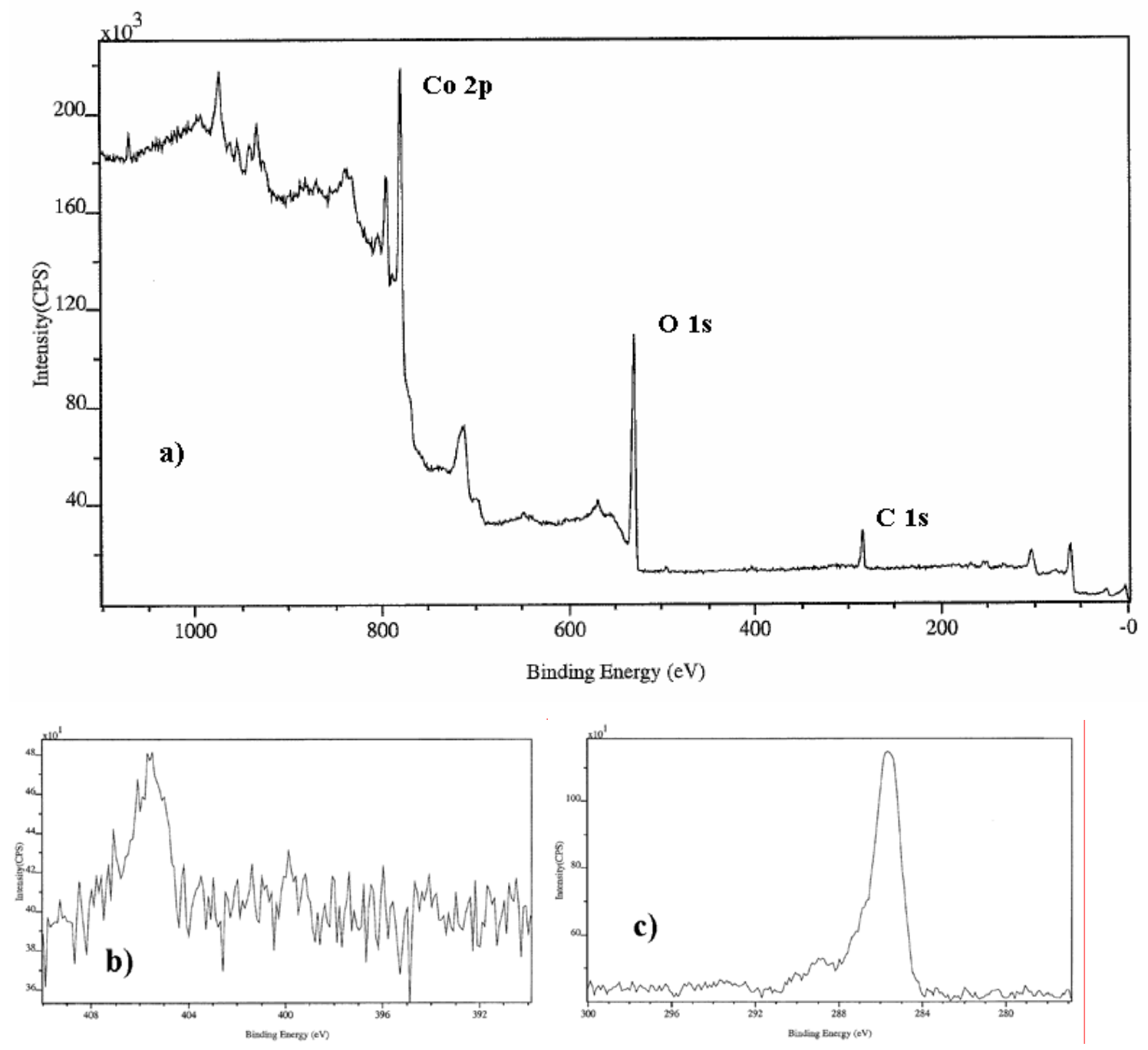

Figure S-4: (a) XPS spectrum of PAN Co nanoparticles after pyrolysis (b) expanded spectrum of nitrogen peaks (c) expanded spectrum of carbon peaks

Solid State Characterization of PAN Co nanoparticles by Raman. Raman measurements for pyrolysized films of PAN and PAN Co nanoparticles were performed on quartz substrates. The pyrolyzed films were prepared from concentrated solutions of PAN or PAN Co nanoparticles in DMF solution $(10 \mathrm{mg} / \mathrm{mL})$. Solutions were drop casting multiple times (four) and allowed to dry 
under argon to ensure sufficiently thick films. The samples were stabilized and pyrolyzed using standard conditions in a tube furnace (Figure S-5).

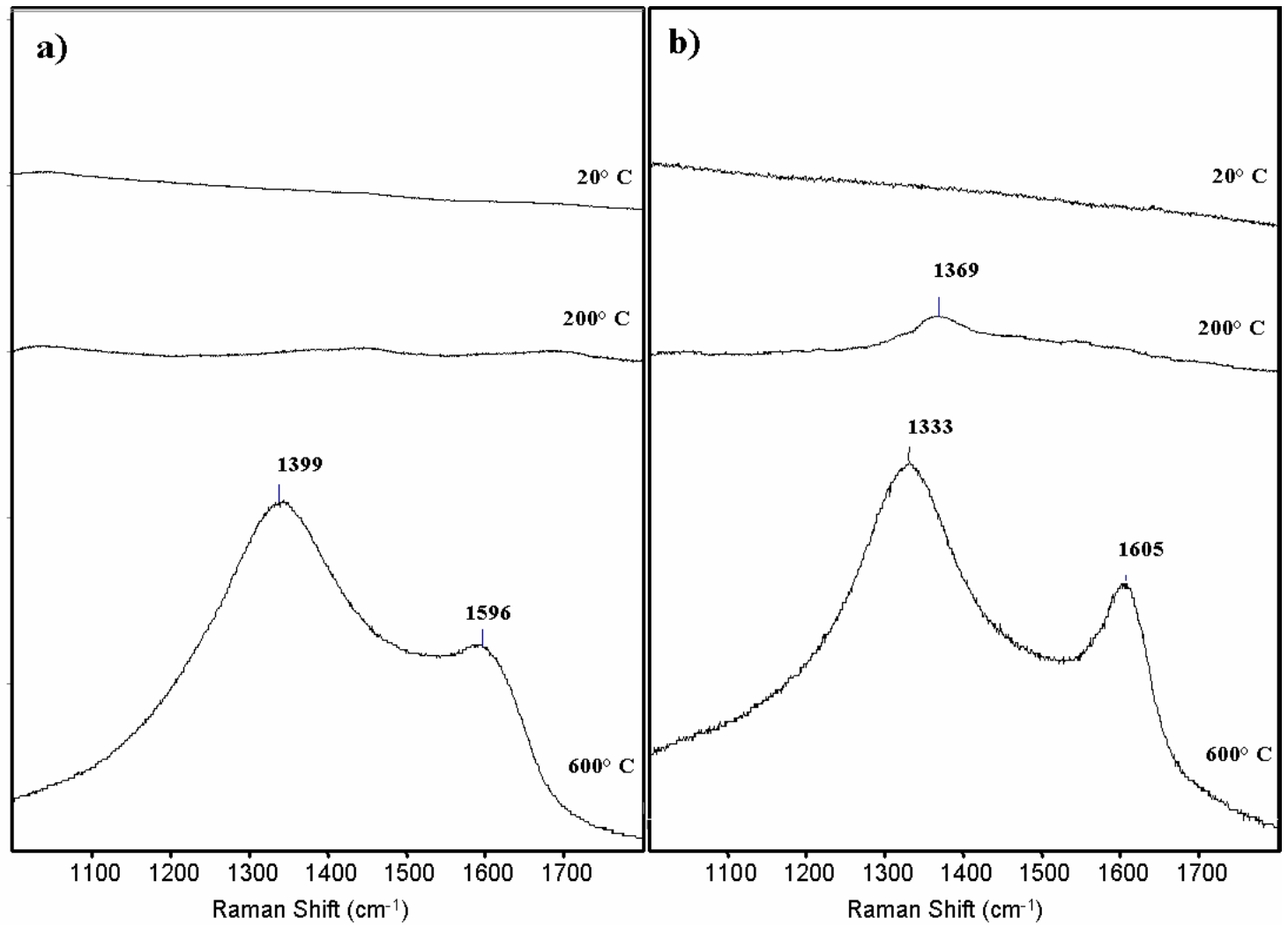

Figure S-5: Bulk Raman spectrum on quartz at various pyrolysis conditions of a) PAN b) PAN-Co nanoparticles.

Raman bands of the room temperature and stabilized PAN and PAN-Co nanoparticles on quartz are very weak due to the amorphous structure of the polymer film, lack of surface enhancement from the quartz substrate, and the presence of an intense background from scattered light of the thick film in relation to the weak Raman modes of PAN. However, Raman measurements after pyrolysis confirm the presence of graphitic (G-band) carbon at 1596 $\mathrm{cm}^{-1}$ for PAN and $1605 \mathrm{~cm}^{-1}$ for the PAN Co nanoparticle composite. The Raman mode for pyrolyzed PAN Co NP at G-band was more intense and sharp than the pyrolyzed PAN sample.

\section{AFM/FESEM/TEM Images}


Preparation of PAN-Co nanoparticles for TEM imaging and analysis. PAN-Co nanoparticles were dispersed $(0.01 \mathrm{mg} / \mathrm{mL}$ in DMF $)$ and a drop of solution was placed onto silicon wafer and dried under argon purge in a magnetic field. Pyrolysis of the samples was performed using typical conditions by heating the $\mathrm{Si}$ wafers in a tube furnace.
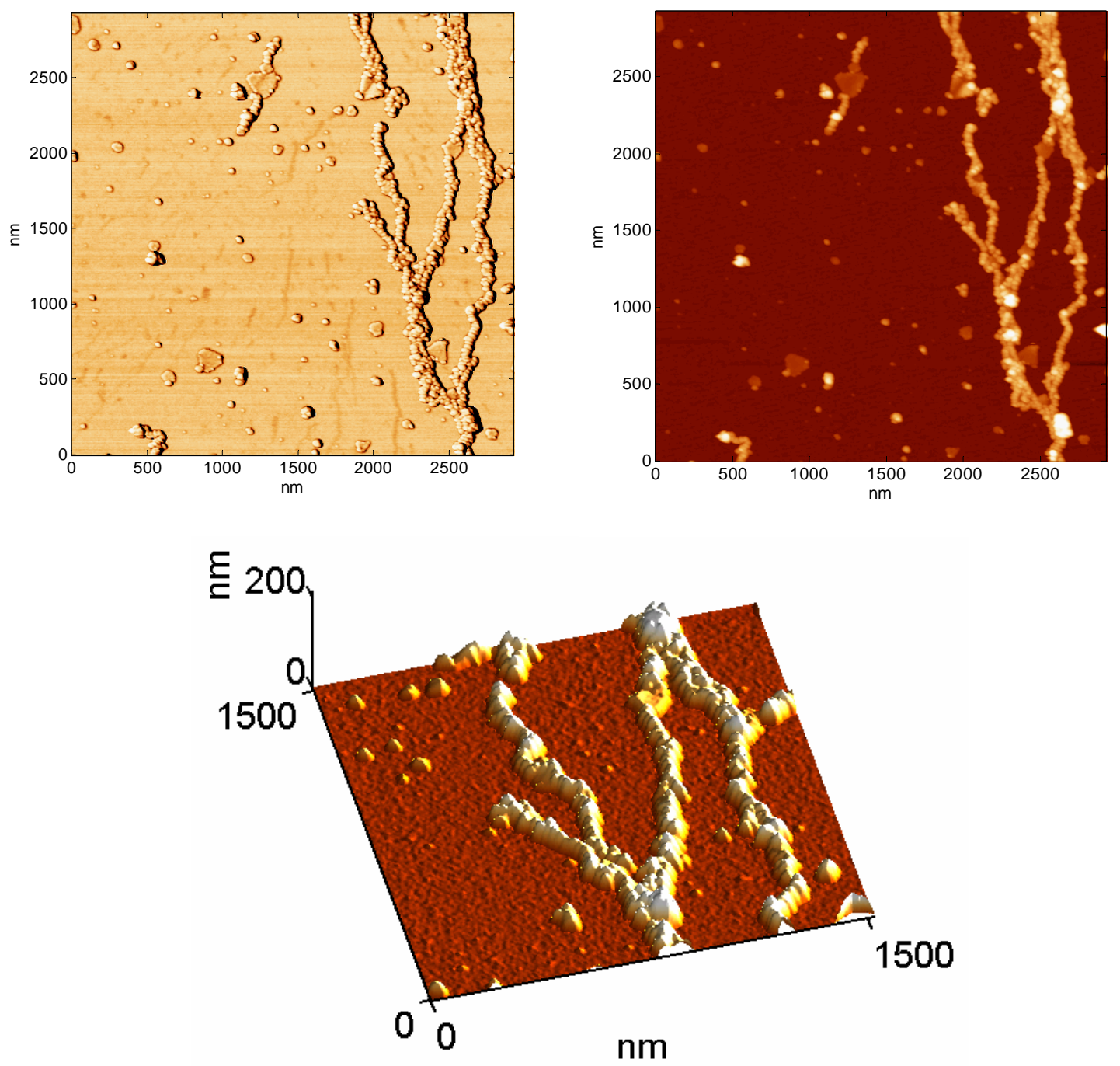

Figure S-6. AFM amplitude (Left), topography (Right), and 3D images (Bottom) of PAN Co nanoparticles cast onto $\mathrm{Si}$ wafer in the presence of external magnetic field before pyrolysis. 

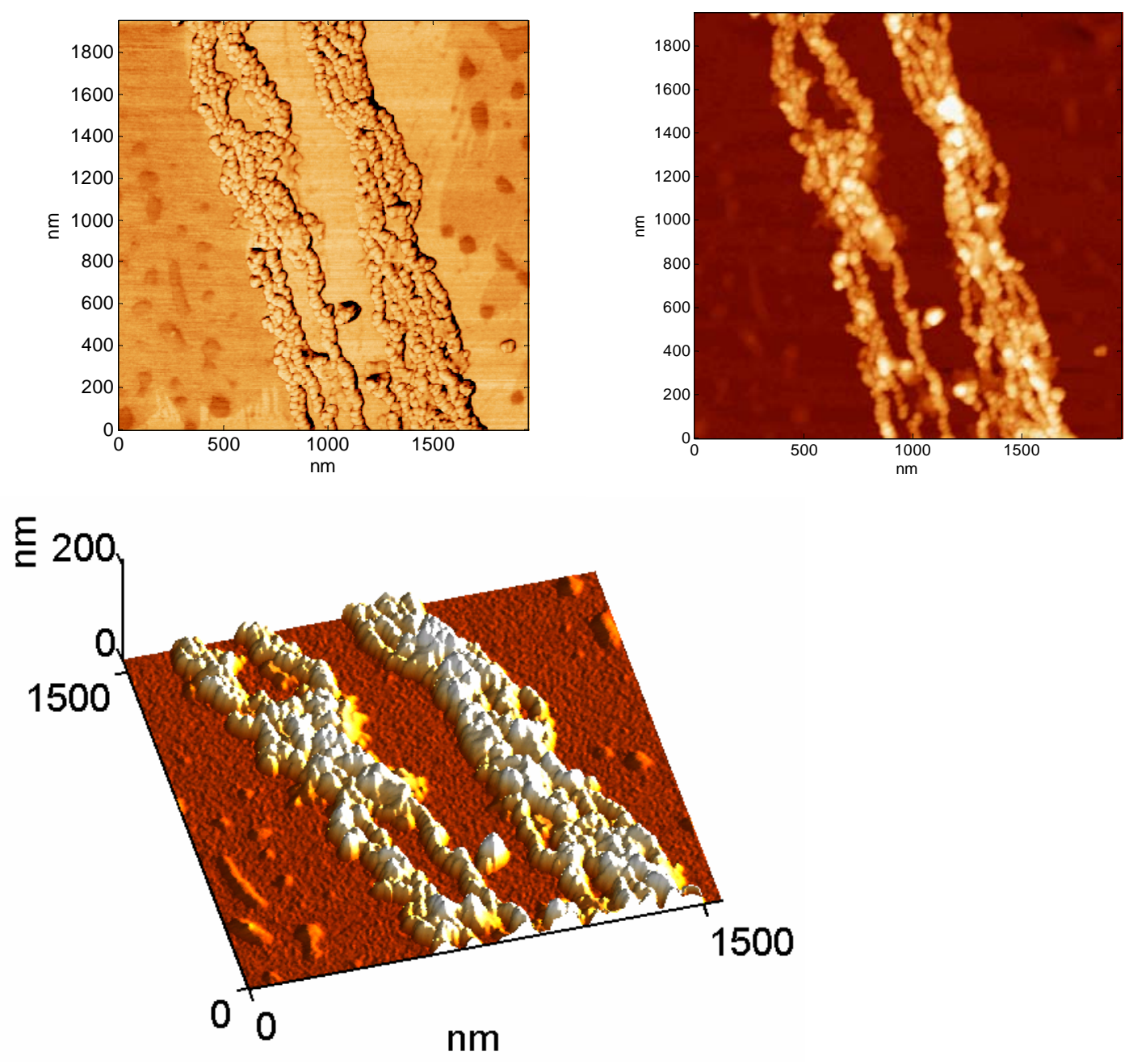

Figure S-7. AFM amplitude (Left), topography (Right), and 3D images (Bottom) of PAN Co nanoparticles cast onto $\mathrm{Si}$ wafer in the presence of external magnetic field after stabilization at $250^{\circ} \mathrm{C}$ in air. 

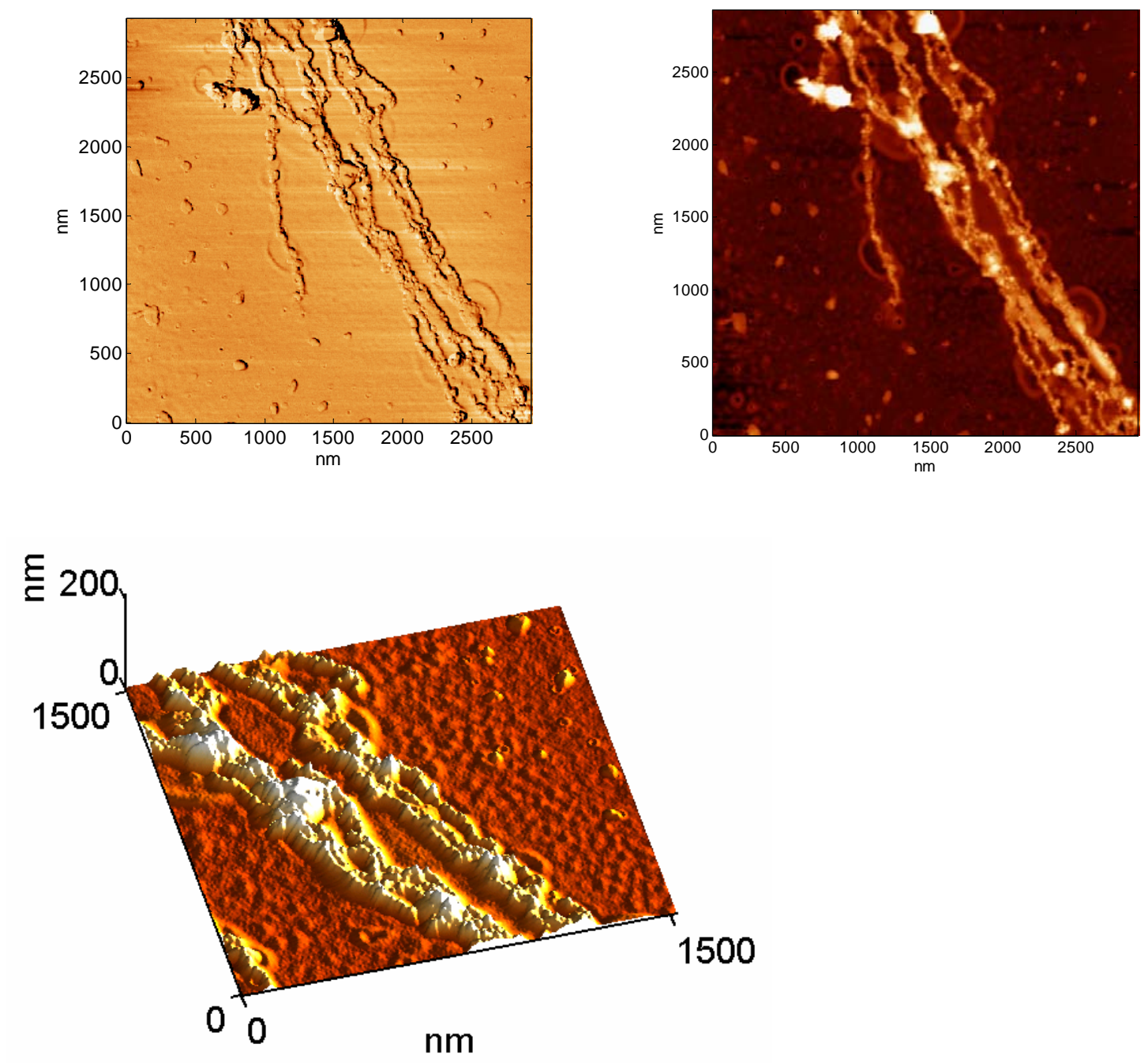

Figure S-8. AFM amplitude (Left), topography (Right), and 3D images (Bottom) of PAN Co nanoparticles cast onto $\mathrm{Si}$ wafer in the presence of external magnetic field after stabilization at $250^{\circ} \mathrm{C}$ in air and pyrolysis at $600^{\circ} \mathrm{C}$ under $\mathrm{N}_{2}$.

The height of the nanoparticles, measured by AFM, was found to decrease with heating during the stabilization and pyrolysis steps. The nanoparticles before pyrolysis were determined to be 28 $\pm 5 \mathrm{~nm}$ tall. This was in reasonable agreement with TEM sizes, with the cobalt core being $18 \mathrm{~nm}$ and 2-3 nm polymer shell. After thermal stabilization at $200^{\circ} \mathrm{C}$ in air, the height of the nanoparticles had decreased to $25 \pm 5 \mathrm{~nm}$, Full pyrolysis at $600^{\circ} \mathrm{C}$ under argon, led to further height decrease to $20 \pm 5 \mathrm{~nm}$. (For particle height distributions see Figures S-9). The height distributions from AFM images are calculated based on 30 particles measurement. This observed 
change was consistent with a decrease in size of polymer shell, corresponding to the loss of mass during the carbonization of PAN.

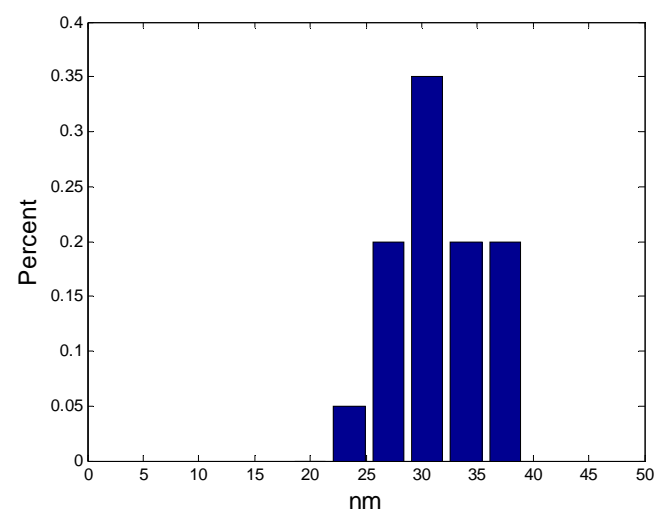

(a)

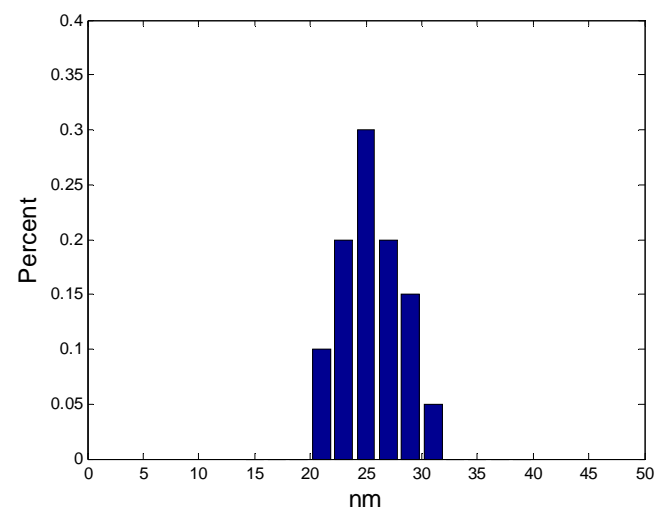

(b)

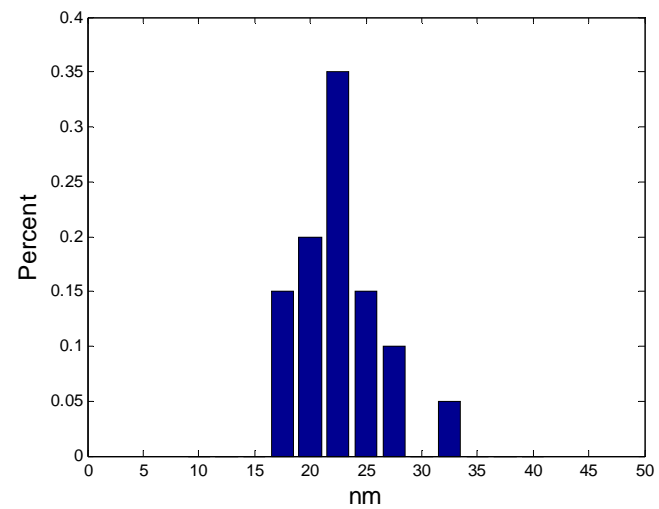

(c)

Figure S-9. Height distributions of PAN Co nanoparticles from AFM height images (a) before pyrolysis (b) after stabilization in air at $250{ }^{\circ} \mathrm{C}$ (c) after stabilization and pyrolysis at $600{ }^{\circ} \mathrm{C}$ in $\mathrm{N}_{2}$. 
Preparation of PAN-Co nanoparticles for TEM imaging and analysis. PAN-Co nanoparticles were dispersed $(0.1 \mathrm{mg} / \mathrm{mL}$ in DMF). A strip of carbon coated mica was lifted onto a droplet of nanopure water followed by lifting of the carbon layer onto a nickel grid. A drop of PAN-Co nanoparticle solution was placed onto the carbon coated nickel grid and allowed to dry. Images of the PAN-Co nanoparticles indicated the cobalt core size $18 \mathrm{~nm}$ and polymer shell 2-3 nm were consistent with previously reported work (Figure S-10).[8] Polymer coated nanoparticles selfassembled into 1-D structures extending over microns long, as observed by TEM. This behavior was observed in the absence and presence of an external magnetic field during drying.

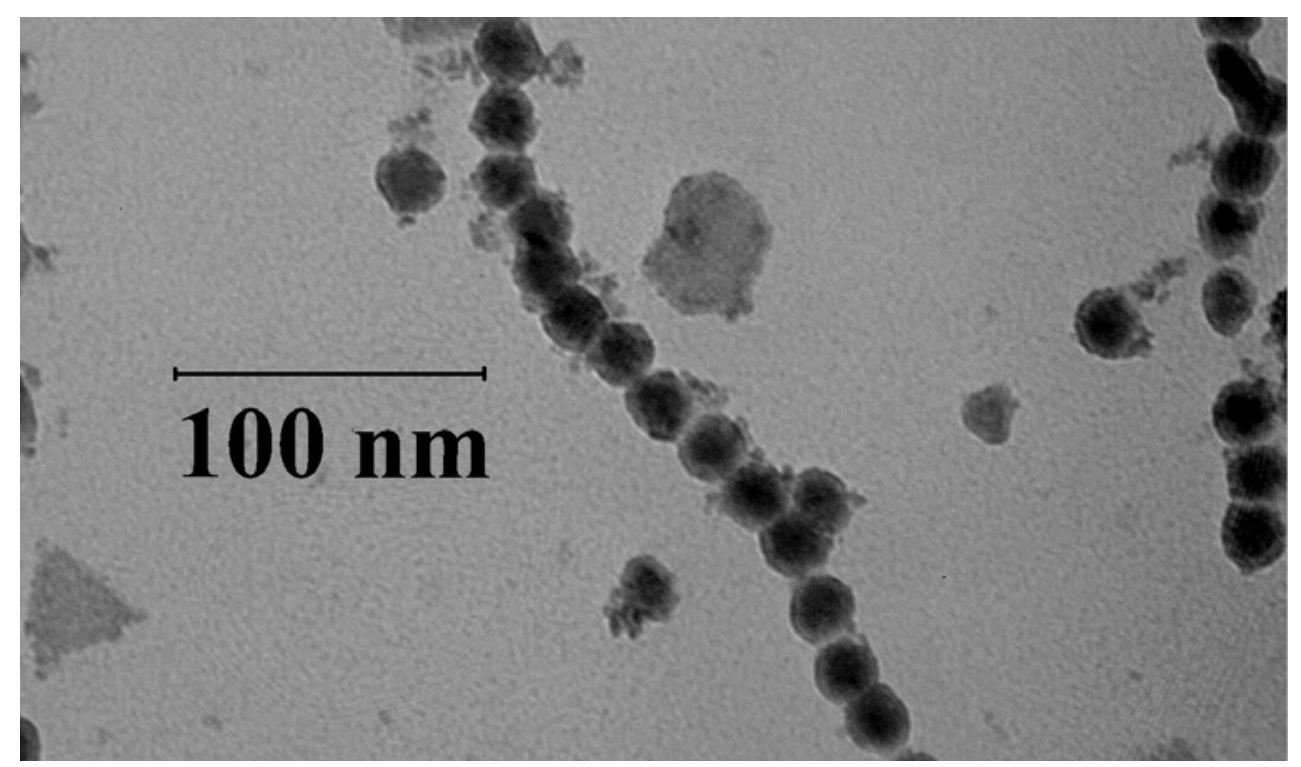

Figure S-10. TEM image of PAN-Co nanoparticles (20 nm) before pyrolysis on a nickel grid.

Preparation of PAN-Co nanoparticles for FE-SEM imaging. PAN-Co nanoparticles were dispersed in organic solvent at two different concentrations: concentrated (10 mg/mL in DMF) and dilute $(0.1 \mathrm{mg} / \mathrm{mL}$ in DMF). Samples were prepared by drop casting the solution onto a silicon wafer and allowed to dry in a magnetic field. FE-SEM imaging of cobalt nanoparticle cores was performed at $5 \mathrm{kV}$, and the images de-phased during the imaging process due to charging. In dilute samples, particles formed 1D chains or clusters of 1D chains, with or without the presence of a magnetic field (Figure S-11,12). In concentrated samples, Co particles formed 2D arrays of chains, with overall arrangement due to the magnetic field (Figure S-13). 


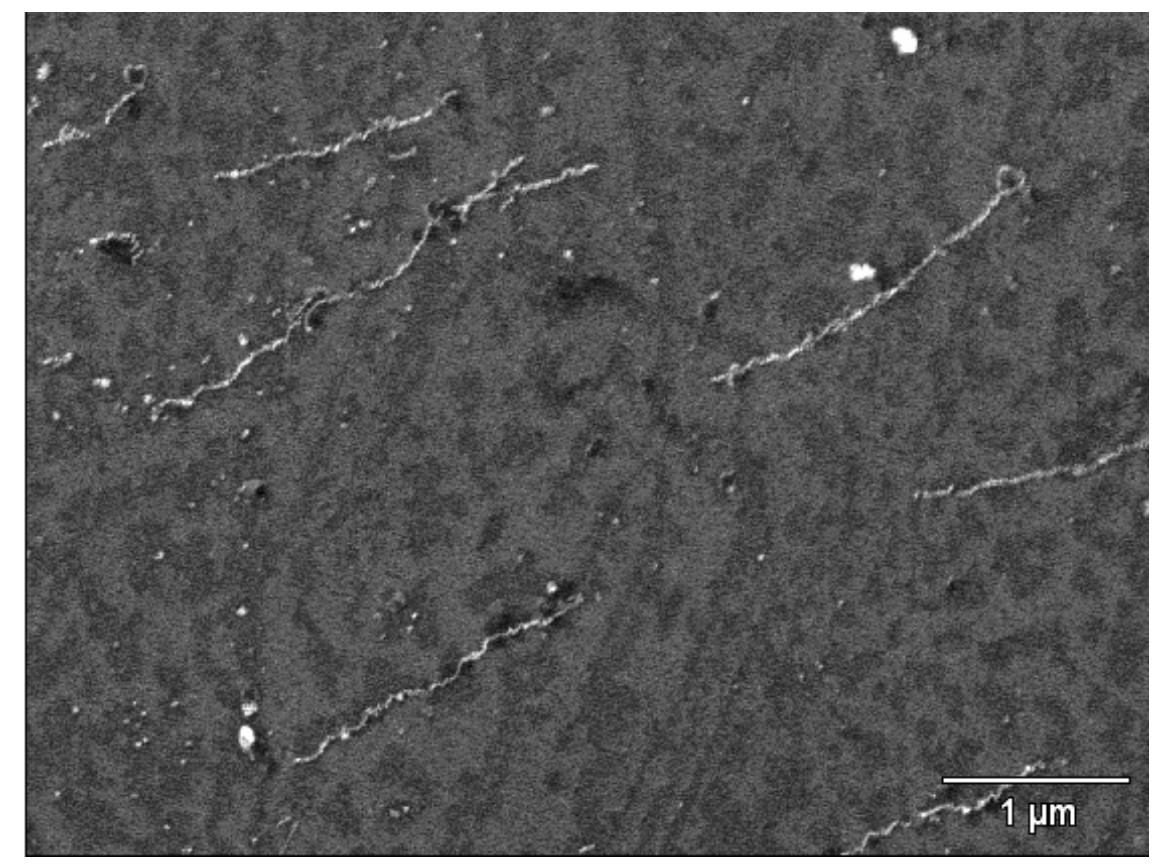

Figure S-11. FESEM image of PAN-Co nanoparticles before pyrolysis on Si wafer..

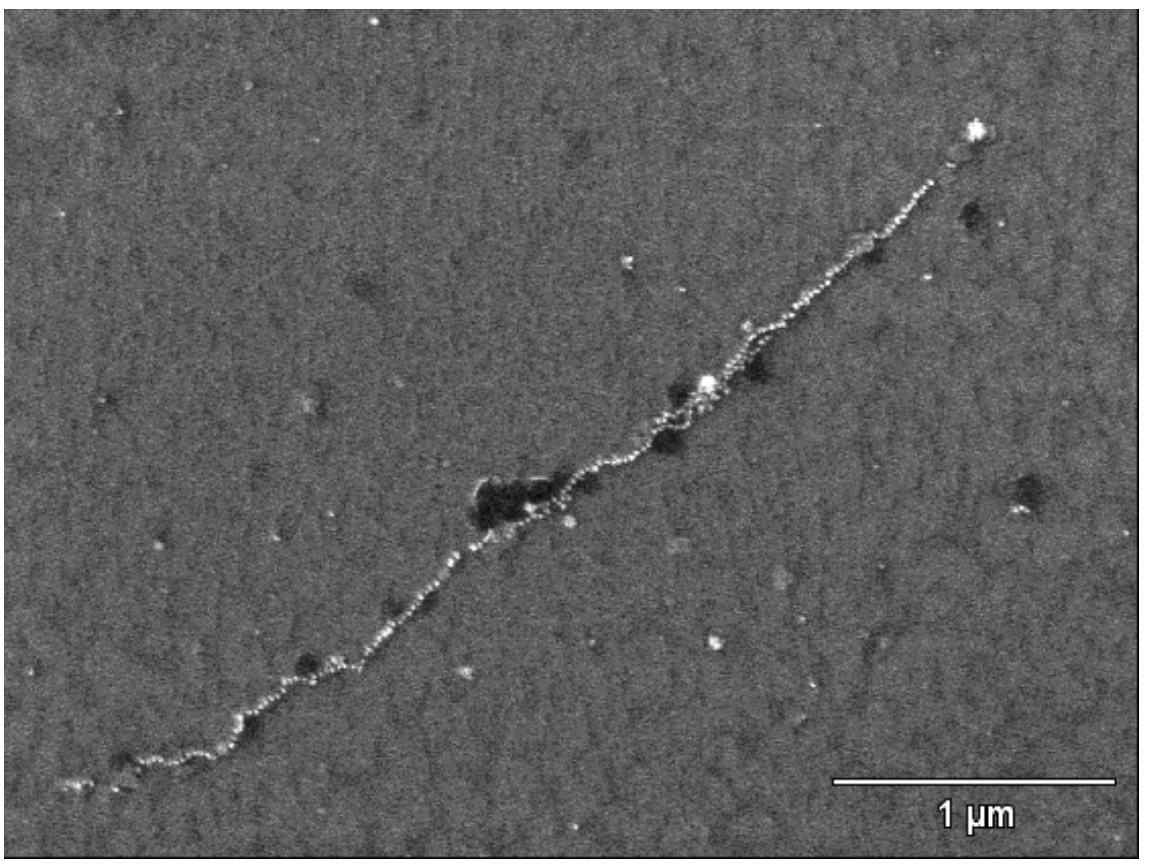

Figure S-12. FESEM image of single chain PAN-Co nanoparticles before pyrolysis on Si wafer. 


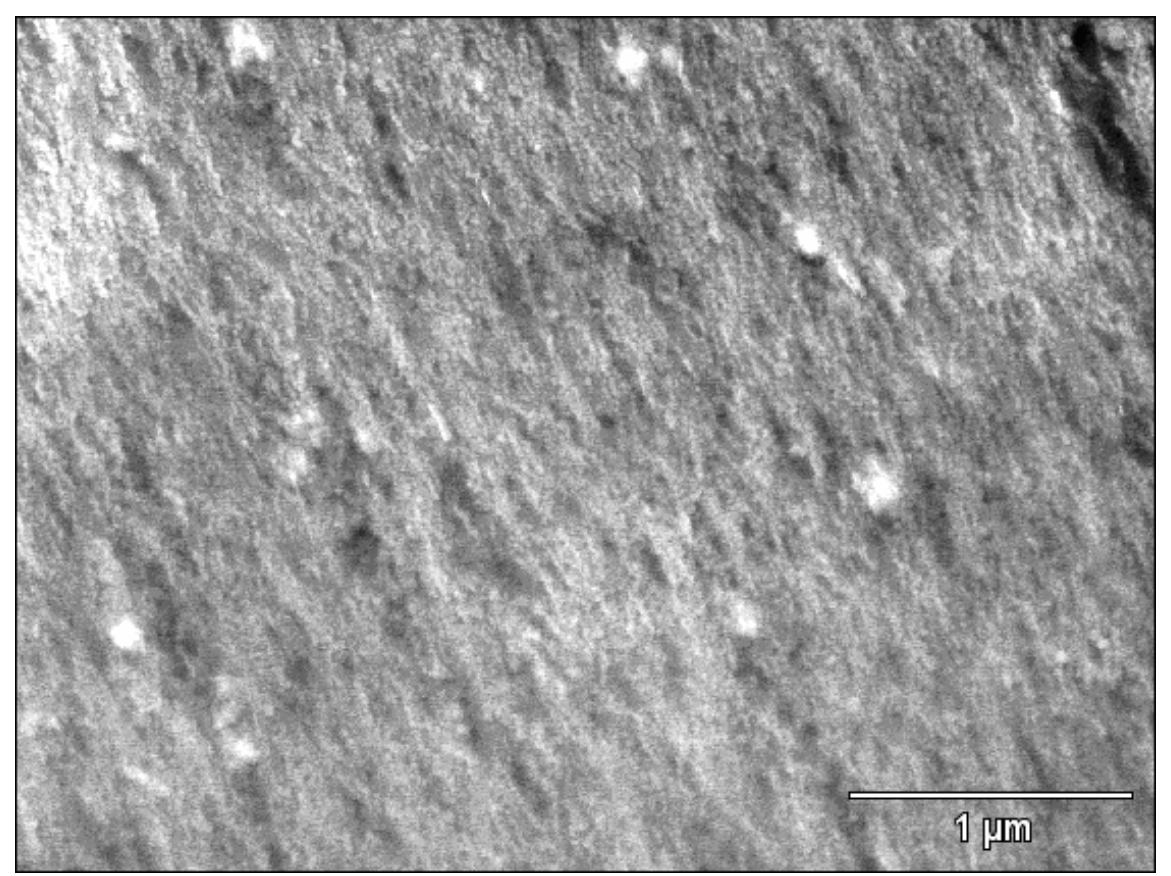

Figure S-13. FESEM image of concentrated PAN-Co nanoparticles before pyrolysis on Si wafer.

Pyrolysis of the samples was performed using typical conditions by heating the Si wafers in a TGA. FE-SEM imaging determined that the pyrolyzed samples were conducting, and charging problems associated with the pre-pyrolyzed samples were not observed. In addition, a larger range of imaging voltages could be used, ranging from $1 \mathrm{kV}$ to $10 \mathrm{kV}$. The change in conductive properties was consistent with pyrolysis of insulating PAN to semiconducting/conducting graphitic carbon. In dilute samples, particles maintained 1D chains or clusters of 1D chains (Figure S-14,15) after carbonization. In concentrated samples, the preservation of alignment of chains was observed after pyrolysis (Figure S-16). 


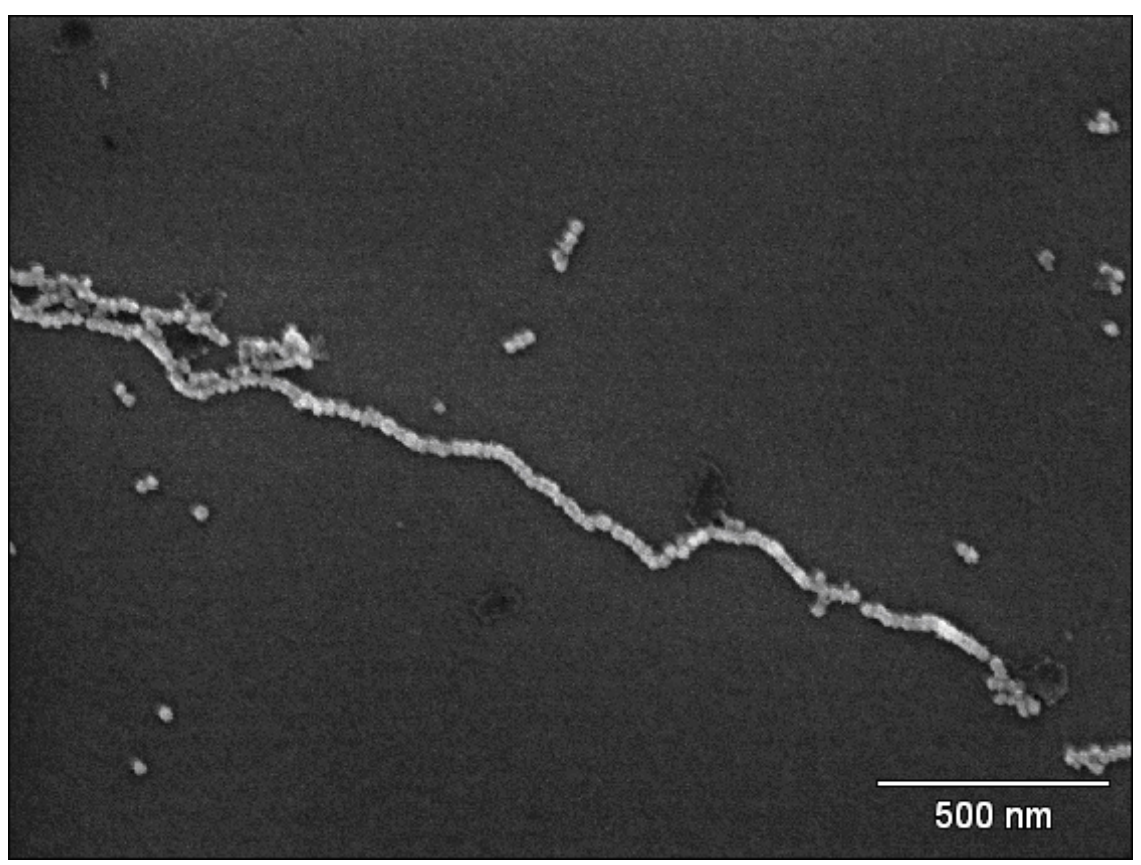

Figure S-14. FESEM image of single chain PAN-Co nanoparticles after pyrolysis on Si wafer.

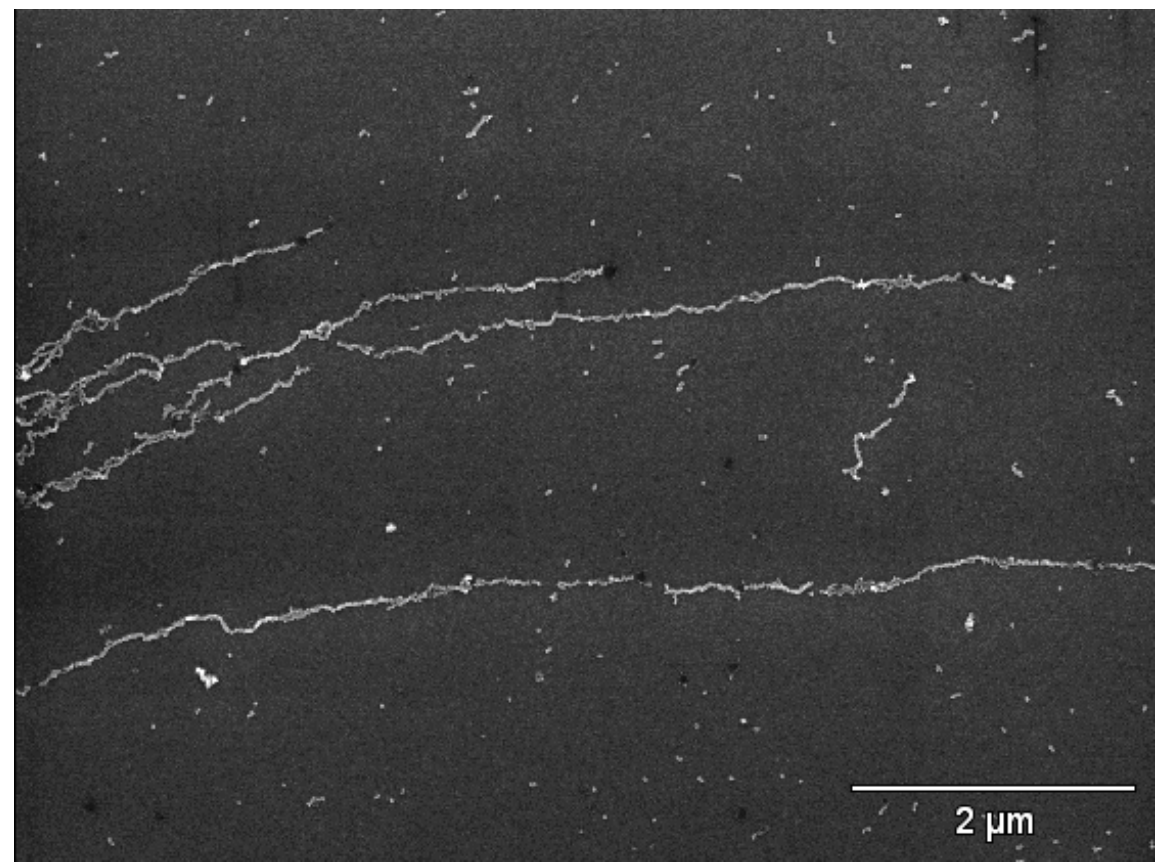

Figure S-15. FESEM image of PAN-Co nanoparticles after pyrolysis on Si wafer. 


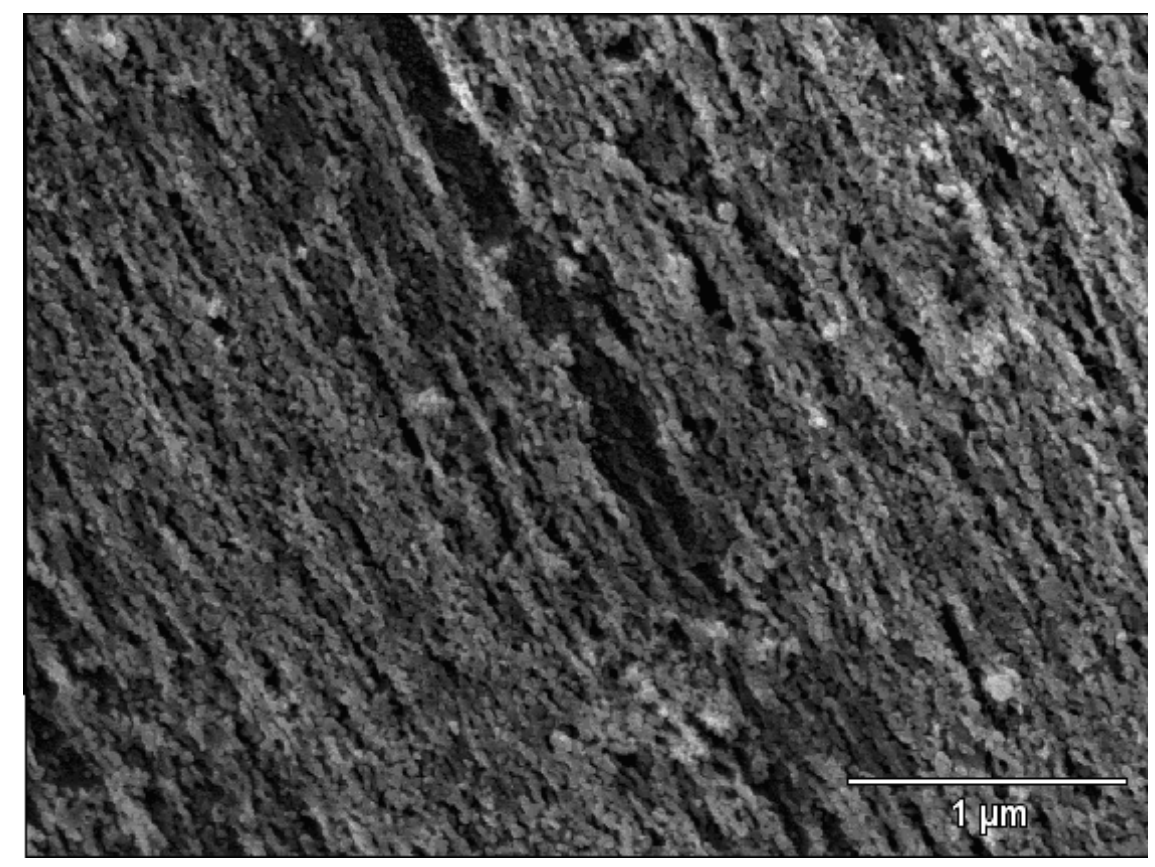

Figure S-16. FESEM image of concentrated PAN-Co nanoparticles after pyrolysis on Si wafer.

\section{Magnetic Properties (VSM)}

Solid state magnetic measurements of PAN-Co nanoparticles by VSM. The magnetic properties of precipitated PAN-Co nanoparticles $(20.0 \mathrm{mg})$ were measured using VSM measurements at $43 \mathrm{~K}$ and $300 \mathrm{~K}$. The PAN-Co nanoparticle powder $\left(\mathrm{M}_{\mathrm{s}}=22.1 \mathrm{emu} / \mathrm{g}\right)$ exhibited ferromagnetic behavior at room temperature $(300 \mathrm{~K})$ with a coercivity $\left(\mathrm{H}_{\mathrm{c}}\right)$ of $254.6 \mathrm{Oe}$, while measurement at low temperature $(40 \mathrm{~K})$ showed an increased coercivity of 1666 Oe and constant $\mathrm{M}_{\mathrm{s}}(22.3 \mathrm{emu} / \mathrm{g})$ (Figure S-17). This was consistent with the magnetic properties of previous pre-exchanged PS-Co particles. [8] 


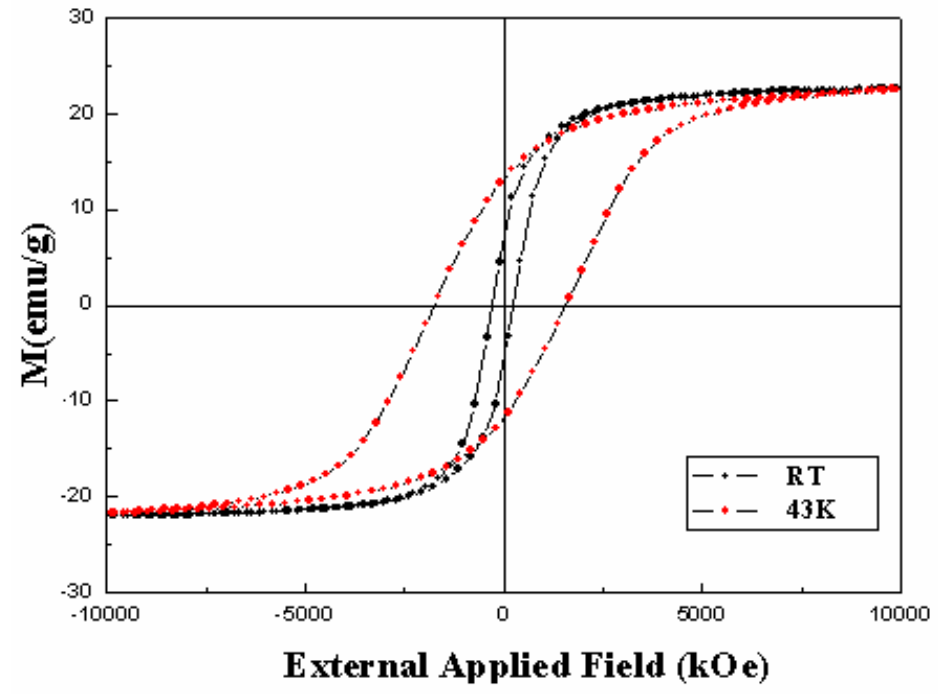

Figure S-17. VSM spectra of PAN-Co nanoparticle powder before pyrolysis.

Pyrolysis was performed on a bulk solid sample (17.6 mg after pyrolysis) in a tube furnace under typical pyrolysis conditions. The pyrolysized PAN-Co nanoparticle powder $\left(\mathrm{M}_{\mathrm{s}}=52.7 \mathrm{emu} / \mathrm{g}\right)$ exhibited ferromagnetic behavior at room temperature $(300 \mathrm{~K})$ with a coercivity $\left(\mathrm{H}_{\mathrm{c}}\right)$ of $836.9 \mathrm{Oe}$, while measurement at low temperature $(40 \mathrm{~K})$ showed an increased coercivity of 1308 Oe and constant $\mathrm{M}_{\mathrm{s}}(53.7 \mathrm{emu} / \mathrm{g}$ ) (Figure S-18). The pyrolysis of the PAN cobalt nanoparticles increased both the coercivity and $\mathrm{M}_{\mathrm{s}}$. XRD of the PAN cobalt nanoparticles showed that the crystal phase of the cobalt had not changed substantially from fcc phase to hcp phase. The increase in coercivity was consistent with annealing of the cobalt inorganic core to a higher degree of crystalinity due to the high temperatures involved in pyrolysis.

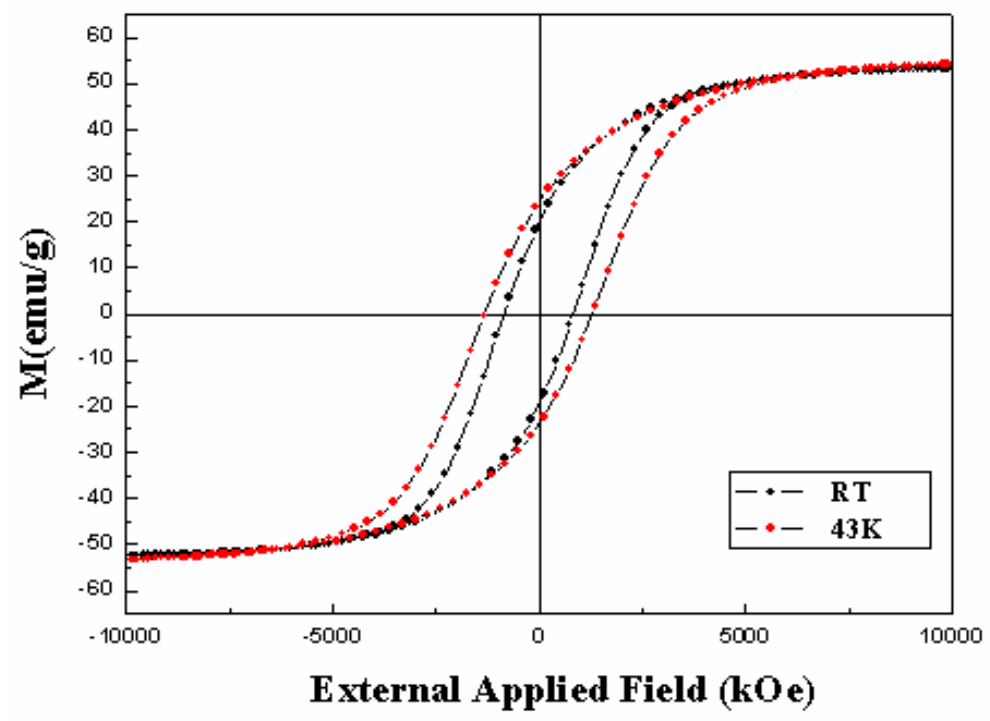

Figure S-18. VSM spectra of PAN-Co nanoparticle powder after pyrolysis. 


\section{References}

1. Fauconnier, N.P., J. N.; Roger, J.; Bee, A., Thiolation of maghemite nanoparticles by dimercaptosuccinic acid. . J. Colloid Interface Sci., 1997. 194 (2): p. 427-433.

2. Kataby, G.C., M.; Prozorov, R.; Gedanken, A., , Coating Carboxylic Acids on Amorphous Iron Nanoparticles. . Langmuir, 1999. 15 (5): p. 1703-1708.

3. Hong, R.F., N. O.; Emrick, T.; Rotello, V. M., Surface PEGylation and Ligand Exchange Chemistry of FePt Nanoparticles for Biological Applications. Chem. Mater., 2005. 17 (18): p. 4617-4621.

4. $\quad$ Thomas, J.R., J. Appl. Phys., 1965. 37: p. 2914-2915.

5. Vadala, M.L.R., M.; Zalich, M. A.; St. Pierre, T. G.; Riffle, J. S., Block copolysiloxanes and their complexation with cobalt nanoparticles. Polymer, 2004. 45(22): p. 7449-7461.

6. Baranauskas, V.V., III; Zalich, M. A.; Saunders, M.; St. Pierre, T. G.; Riffle, J. S., Poly(styrene-b-4-vinylphenoxyphthalonitrile)-Cobalt Complexes and Their Conversion to Oxidatively Stable Cobalt Nanoparticles. Chemistry of Materials, 2005. 17(21): p. 5246-5254.

7. Matyjaszewski, K.J.S.M.P.H.J.G., S.G., Synthesis of Well-Defined Polyacrylonitrile by Atom-Transfer Radical Polymerization. Macromolecules, 1997. 30: p. 6398-6400.

8. Korth, B.D.K., P.; Shim, I.; Bowles, S.E.; Tang, C.; Kowalewski,T.; Nebesny, K.W.; Pyun, J., Polymer Coated Ferromagnetic Colloids from Well-Defined Macromolecular Surfactants and Assembly into Nanoparticle Chains. J. Am. Chem. Soc., 2006. 128(20): p. 6562-6563.

9. Leiston-Belanger, J.M.P., J. ; Russell, T. P., Synthesis and microphase separation of poly(styrene-b-acrylonitrile) prepared by sequential anionic and ATRP techniques. Macromolecules, 2006. 39: p. 1766-1770.

10. Puntes, V.F.Z., D.; Erdonmez, C. K.; Alivisatos, A. P., J. Am. Chem. Soc., 2002. 124: p. 12874-12880.

11. Weidenthaler, C.L., A.H.; Schmidt, W.; Schuth, F., X-ray photoelectron spectroscopic studies of PAN-based ordered mesoporous carbons (OMC). Microporous \& Mesoporous Materials., 2006. 88(1-3): p. 238-243.

12. Tomita, S.H., M.; Fujii, M.; Hayashi, S.; Akamatsu, K.; Deki, S.; Yasuda, H., Formation of Co filled carbon nanocapsules by metal-template graphitization of diamond nanoparticles. J. Appl. Phys., 2000. 88 (9): p. $5452-5456$ 